\title{
NANCY NATURAL RADIOCARBON MEASUREMENTS V
}

\author{
R COPPENS, B GUILLET, R JAEGY and P RICHARD
}

Laboratoire de Radiogéologie. Ecole Nationale Supérieure de Géologie BP 452, 54001 NANCY, France

The following list includes some measurements made during 19751978 since our last list ( $R, 1978, v 20, p$ 62-67) in the National Radiocarbon Laboratory of the Ecole Nationale Supérieure de Géologie Appliquée et de Prospection Minière (ENSG) de Nancy.

Laboratory procedures and techniques are as reported in Hassko et al (1974) where the sample is synthesized to benzene. The only difference is in the counting equipment. We now use a Packard Tricarb 3305 liquid scintillation spectrometer where samples are counted for 24 or 48 hours. Radiocarbon ages are calculated using ${ }^{14} \mathrm{C}$ half-life of 5568 years and $95 \%$ activity of NBS oxalic acid is used as modern standard. Anthracite coal and Merck commercial benzene are used for the dead carbon run.

Counting errors are expressed at $1 \sigma$ confidence level. $\mathrm{AD} / \mathrm{BC}^{*}$ dates are corrected using ${ }^{14} \mathrm{C}$ half-life of 5730 years and according to the Masca correction curve (Ralph et al, 1973).

Description and comments are generally based on information supplied by those who submitted the samples.

\section{SAMPLE DESCRIPTIONS}

I. ARCHAEOLOGIC AND HISTORIC SAMPLES

\section{A. Peru}

The following samples from different prehistoric villages in Peru are a continuation of the series previously described (R, 1978, v 20, p 6267). Coll and subm by Frederic Engel, Dir Mission archaeol française au Pérou and Inst Antropol y Agric Precolombina, Univ Nac Agraria, Lima, Peru.

\section{Chillon program series}

Sites with Neolithic IV or $V$ pottery

\section{Ny-367. Chillon valley, $\mathrm{V} 3563$}

$2060 \pm 70$

Plant material from Village $11 \mathrm{~b}$ X-131, Chacra alta Layer 100, alt $15 \mathrm{~m}\left(11^{\circ} 56^{\prime} 00^{\prime \prime} \mathrm{S}, 77^{\circ} 05^{\prime} 00^{\prime \prime} \mathrm{W}\right)$. Comment (FE): dates a post-Chavin, pre-early Lima settlement in the Chillon valley. $C f$ Willey's white on red coll 1973 by F Engel and B Ojeda.

\section{Southern deserts series}

1. Preagricultural sites

\section{Pampa Colorado Fog oasis series}

Ny-383. Fog oasis V 3653

Charcoal from Encampment 17 c VIII-280A, Layer 400, alt 30 to $50 \mathrm{~m}$ $\left(16^{\circ} 31^{\prime} 30^{\prime \prime} \mathrm{S}, 72^{\circ} 51^{\prime} 30^{\prime \prime} \mathrm{W}\right)$. Comment (FE): could be one of oldest 
Holocene settlements known in deep south of Peru. Located in fog oasis extending to bluff overhanging ocean. Estimated age: 9000 вр. Coll 1975 by $\mathrm{F}$ Engel.

Ny-381. Fog oasis V 3651

$9310 \pm 120$

Shells from Encampment 18 b IX-5, Las Higueras, alt $10 \mathrm{~m},\left(16^{\circ} 14^{\prime}\right.$ $\left.20^{\prime \prime} \mathrm{S}, 71^{\circ} 34^{\prime} 57^{\prime \prime} \mathrm{W}\right)$. Comment $(\mathrm{FE})$ : will date 1 of early Holocene encampments found on bluff overhanging ocean. Coll 1975 by $\mathrm{F}$ Engel.

\section{Ny-387. Fog oasis V 3657}

$\mathbf{8 4 9 0} \pm \mathbf{1 5 0}$

Shells from Encampment 17 c VIII-2015, El Carrizal, alt $200 \mathrm{~m}\left(16^{\circ}\right.$ $29^{\prime} \mathrm{S}, 72^{\circ} 55^{\prime} \mathrm{W}$, central point). Comment (FE): will date early settlement in Pampa Colorado fog oasis. Coll 1975 by F Engel.

Ny-386. Fog oasis, V 3656

$6620 \pm 100$

Shell from Encampment 19 a V-300, Punta Icuy, Layer 200, alt 25m $\left(17^{\circ} 49^{\prime} 00^{\prime \prime} \mathrm{S}, 71^{\circ} 08^{\prime} 20^{\prime \prime} \mathrm{W}\right)$. Comment (FE): will help date encampments found on bluff overhanging ocean. Coll 1975 by F Engel.

\section{Pottery-yielding settlements, Neolithic}

\section{Ny-380. Fog oasis, V 3650}

$3510 \pm 80$

Charcoal from very large, La Buitrera village, 19 a II-100, from foot of fog oasis in marshy area. Alt $150 \mathrm{~m}\left(17^{\circ} 45^{\prime} \mathrm{S}, 71^{\circ} 10^{\prime} \mathrm{W}\right.$, central area). Comment (FE): La Buitrera was occupied at least twice, first during preforming days. Sample is from Layer 200, typologically showing early occupation by settlers using pottery. Coll 1975 by F Engel.

\section{Pottery yielding settlements, alloyed metal period}

\section{Ny-384. Fog oasis, $\mathrm{V} 3654$ \\ $1100 \pm 100$}

Bones from Village $19 \mathrm{~b}$ VII-105, Loma el Platanillo, Layer $100\left(18^{\circ}\right.$ $02^{\prime} 25^{\prime \prime} \mathrm{S}, 76^{\circ} 46^{\prime} 00^{\prime \prime} \mathrm{W}$ ) alt $270 \mathrm{~m}$. Comment (FE): correct date for Tiahuaneo influenced village would read 1200 to 900 BP, AD 750 to 1050 .

\section{Ny-358. Fog oasis}

$\mathbf{8 7 0} \pm 80$

Village at mouth of Ica R; raft made of reeds was found hidden in sand dune. Some reeds were used for dating. Alt $20 \mathrm{~m}\left(14^{\circ} 52^{\prime} 00^{\prime \prime} \mathrm{S}, 75^{\circ}\right.$ $33^{\prime} 34^{\prime \prime}$ W). Comment (FE): raft was decorated with fragments of shells (Spondylus sp), typical Tiahunacoid cultural trait in Peru (only Precolombian raft known in South America, now in Mus Nat Agrarian Univ, Lima). Coll 1974 by F Engel.

\section{Ny-385. Fog oasis, V 3655}

AD 1300*

$650 \pm 70$

Bones from Encampment 17 c VII-715, Palo Parado, on bluff. Alt 75 to $100 \mathrm{~m}$. Stone bldg ( $16^{\circ} 29^{\prime} 00^{\prime \prime} \mathrm{S}, 73^{\circ} 03^{\prime} 00^{*} \mathrm{~W}$ central point). Comment (FE): could be late Pre-Incaic. Coll 1975 by F Engel. 


\section{Central coast program series}

1. Preceramic horizons

\section{Ny-414. Fog oasis, $\mathrm{V} 3704$}

$4885 \pm 90$

Charcoal from Village 13 a II-90, Quilmana I, Layer 200. Alt $440 \mathrm{~m}$ (12 $\left.53^{\prime} 58^{\prime \prime}, 76^{\circ} 26^{\prime} 03^{\prime \prime}\right)$. Comment (FE): will help date early farming settlements on central coast. Coll 1976 by B Ojeda.

Ny-415. Fog oasis, $\mathrm{V} 3708$

$4250 \pm 70$

Charcoal from Village 12 b XI-500, Omaniso I, Layer 100, alt $440 \mathrm{~m}$ $\left(12^{\circ} 57^{\prime} 20^{\prime \prime} \mathrm{S}, 76^{\circ} 27^{\prime} 54^{\prime \prime}\right)$. Comment (FE): same as Ny-414. Coll 1976 by B Ojeda.

\section{Ny-418. Fog oasis, V 3757}

$3580 \pm 80$

Charcoal from Village 13 a II-45, Carretilla, Layer 200 , alt $150 \mathrm{~m}\left(12^{\circ}\right.$ $\left.58^{\prime} 26^{\prime \prime} \mathrm{S}, 76^{\circ} 27^{\prime} 56^{\prime \prime} \mathrm{W}\right)$. Comment (FE): same as Ny-414. Coll 1976 by B Ojeda.

\section{Ny-416. Fog oasis, V 3709}

$3550 \pm 80$

Charcoal from Village 13 a II-70, Quilmana II, Layer 100, alt $350 \mathrm{~m}$ $\left(12^{\circ} 55^{\prime} 30^{\prime \prime} \mathrm{S}, 76^{\circ} 26^{\prime} 45^{\prime \prime} \mathrm{W}\right)$. Comment (FE): same as Ny-414. Coll 1976 by B Ojeda.

\section{Ny-417. Fog oasis, V 3750}

$3430 \pm 80$ $\mathbf{5 3 8 0 *}$

Charcoal from Village 13 a II-10, Quilmana III, Layer 100 , alt $310 \mathrm{~m}$ $\left(12^{\circ} 56^{\prime} 50^{\prime \prime} \mathrm{S}, 76^{\circ} 26^{\prime} 42^{\prime \prime} \mathrm{W}\right)$. Comment (FE): same as Ny-414. Coll 1976 by B Ojeda.

\section{Ny-419. Fog oasis, $\mathrm{V} 3760$}

$3240 \pm 80$

Charcoal from Village 13 a II-20, Cerro Grande, Layer 200, alt $330 \mathrm{~m}$

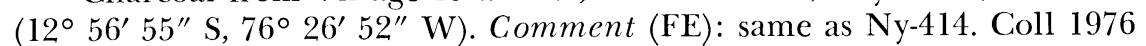
by B Ojeda.

\section{Ny-392. Fog oasis, V 3700}

$4740 \pm 100$

Carbonized wood fragment from village 12 b IV-490 $\left(12^{\circ} 20^{\prime} 30^{\prime \prime} \mathrm{S}\right.$, $76^{\circ} 43^{\prime \prime} \mathrm{W}$ ). Los Icasos, alt $280 \mathrm{~m}$. Comment (FE): estimated age: 5000 вр?. Could be a coastal Neolithic I village. Coll 1975 by F Engel.

\section{Ny-393. Fog oasis, V 3702}

$3965 \pm 100$

Structure seems to be related to late preceramic or Neolithic III without maize. It seems to be a common structure which is very rare at that time. Estimated age: 3500 вр. Coll 1975 by F Engel. 
2. Alloyed metal periods

\section{Ny-477. V 6170 A}

$680 \pm 75$

AD 1275*

Fragment of beam supporting roof of stone house, Village $12 \mathrm{~b}$ II-15, Orcocoto, Lurin Valley, alt $2070 \mathrm{~m}\left(12^{\circ} 06^{\prime} 40^{\prime \prime} \mathrm{S}, 76^{\circ} 27^{\prime} 00^{\prime \prime} \mathrm{W}\right)$. Comment (FE): will help date "Cuculi period" villages of 13th-14th centuries AD. Coll 1977 by F Engel.

\section{B. Ivory Coast}

Samples were dated to study history of Kingdom of Kong, commercial area in savannah between forest and Sahel, Sahara and North Africa.

\section{Kong (Ferkessidougou Dept) series} $\left(9^{\circ} 10^{\prime} \mathrm{N}, 4^{\circ} 30^{\prime} \mathrm{W}\right)$

Ny-395. Kong, Site 5000

$$
\mathbf{3 5 0} \pm \mathbf{1 2 0}
$$

Estimated age: 1400 to 1800 . Coll 1975 by V Diabaté Tiégré.

Ny-405. Kong, Site 6000

$195 \pm 85$

\section{AD 1650*}

Charcoal coll at $210 \mathrm{~cm}$ below surface. Estimated age: AD 1400 to 1800 . Coll 1976 by V Diabaté Tiégré.

\section{Ny-406. Kong, Site 7000}

Charcoal coll at $130 \mathrm{~cm}$ below surface. Estimated age: AD 1400 to 1800 . Coll 1976 by V Diabaté Tiégré.

Ny-409. Kong, Mosquée de Sitafa

$$
410 \pm 90
$$

Charcoal coll $150 \mathrm{~cm}$ below surface. Estimated age: AD 1400 to 1800 . Coll 1976 by V Diabaté Tiégré.

Ny-410. Kong, Grande Mosquée

$335 \pm 85$

Charcoal coll $60 \mathrm{~cm}$ below surface. Estimated age: AD 1400 to 1800 . Coll 1976 by V Diabaté Tiégré.

\section{Ny-404. Labiné, $2 \mathrm{~km}$ SE Kong, Site 1000}

Charcoal coll $150 \mathrm{~cm}$ below surface. Estimated age: AD 1300 to 1700 . Coll 1976 by V Diabaté Tiégré.

Ny-407. Ténéguéla, 9km Sud Kong $340 \pm 90$

Charcoal coll $180 \mathrm{~cm}$ below surface. Estimated age: AD 1400 to 1800 . Coll 1976 by V Diabaté Tiégré.

Ny-399. Ténéguéla, $9 \mathrm{~km}$ Sud Kong

Humus 15 to $20 \mathrm{~cm}$. Coll $80 \mathrm{~cm}$ below surface. Coll 1975 by J Polet. 
Sud Aby lagoon series Coast.

Samples were dated to establish historic population maps of SE Ivory

Ny-401. Assoco-Mossobaha Island

AD 1740*

Wood fragments from piles $\left(3^{\circ} 30^{\prime} \mathrm{W}, 5^{\circ} 20^{\prime} \mathrm{N}\right)$. Coll $75 \mathrm{~cm}$ below surface. Coll 1975 by J Polet.

Ny-408. Nyamva Island

$2250 \pm 95$

Charcoal under dense forest (10cm humus). Coll 1975 by J Polet $130 \mathrm{~cm}$ below surface.

\section{Shortugaï series}

\section{Afghanistan}

Charcoal from hearths and ash layers. Ash with burned soils (marl) at different stratigraphic levels, coll from Protohistoric site of Shortugaï (NE Afghanistan). Project was undertaken to study relations between Indus Valley and Central Asian civilizations in Bronze age period (Francfort \& Pottier, 1978). Coll by H P Francfort and subm by J C Gardin.

\section{Ny-421. SHB 76, late Period III, Level 4}

$3535 \pm 165$

Estimated age: ca 3450 вр.

\section{Ny-424. SHB 76, Level 3, early Period III}

$4000^{*}$

Estimated age: ca 3650 вр. Sample was very small. Benzene obtained was minimal.

Ny-427. SHB 76, Level 5, early Period III

$3180 \pm 335$ 3480*

Estimated age: ca $3650 \mathrm{BP}$.

Ny-428. SHB 76, Level 5, early Period III $3710 \pm 100$ 4110*

Estimated age: са 3650 вр.

Ny-422. SHB 76, Level 2, Period II

Estimated age: ca 3750 вр.

Ny-429. SHB 76, Level 3, early Period II

$4190 \pm 125$

4875*

$3050 \pm 250$

$3330 *$

Estimated age: ca $3850 \mathrm{BP}$.

$4375 \pm 160$

5106*

Ny.430. SHB 76, Level 1, late Period I

$4075 \pm 95$

$4710^{*}$

Estimated age: ca 3950 вр.

Ny-425. SHB 76, Level 1, early Period I

$4040 \pm 100$

Estimated age: ca 4050 вр.

4605* 
General Comment: Masca corrected dates run generally several centuries older than tentative chronology based on ceramic typology. Discrepancy perhaps is due to contamination of samples by older mineralogic carbon.

\section{France}

\section{Gordes series, Vaucluse, France}

Wood fragments from cellar, estimated age: 15th or 16th century. Coll and subm by $\mathrm{J} L \mathrm{~L}$ Morand.

Ny-473.

Wood from an ancient oil mill.

Ny-541.

Wood from beam in wall of cellar.

Ny-542.

Well-preserved wood from wooden chimney funnel in wall of cellar.

General Comment (RC): beam and chimney funnel seem to be same age, ca AD 1600, late 16th or early 17th century, and suggest redisposition of cellar. Oil mill seems older, 15 th century. Either is truly older or corresponds to re-use of old wood in new structure.

\section{Ny-485. Limoges, Haute Vienne, rue du Clos Adrien $\begin{array}{r}1980 \pm 80 \\ 30\end{array}$}

Charcoal from Gallo Roman well with pottery and tegulae, of 2nd century. Coll 1977 by J P Loustaud and subm by J M Desbordes. Estimated age: 2nd century. Comment: date appears older than expected based on historic correlations, but wood may be really older.

\section{Ny-498. Roman church of Meymac, Corrèze}

$770 \pm 75$

Charcoal from glass-founder furnace in chancel of church. Sample No. 2 c/III. Durst hole furnace No. 2. Coll and subm by J M Desbordes. Estimated age: 12th century.

\section{Ny-497. Roman church of Meymac, Corrèze}

$$
265 \pm 75
$$

AD 1570*

Charcoal from site below choir of church in Roman surroundings. Coll 1978 and subm by J M Desbordes. Estimated age: 12th century.

\section{Ny-499. Tarnac, Corrèze}

$2020 \pm 70$

Finely dispersed charcoal from base of funeral mound embedded in brown and tamped soil. Coll 1977 and subm by J M Desbordes. Estimated age: 12th century Iron age. Comment: date is younger than expected. Sample does not belong to Prehistoric occupation or may be contaminated by rootlets or humic acids. 
Ny-486. Jarnages, Creuse, lieu-dit Martinfort

Carbonized wood from early Gallo-Roman site with pottery and tiles. Coll 1977 by M Bord and subm by J M Desbordes. Estimated age: 1st century.

Ny-489. Razes, Haute-Vienne, lieu-dit Augères AD 1305*

$660 \pm 80$

Carbonized wood from fill of underground gallery with post GalloRoman pottery. Coll 1977 by R Saumande and subm by J M Desbordes. Estimated age: post-Gallo-Roman period.

Ny-488. Saint Pardoux, Haute Vienne, lieu-dit La Ribière

$600 \pm 90$

AD 1380*

Carbonized wood from fill of underground gallery with post-GalloRoman pottery. Coll 1977 by R Saumande and subm by J M Desbordes. Estimated age: post-Gallo-Roman period.

\section{Ny-487. Cloister of Obazine, Corrèze}

$960 \pm 65$

Wood fragment from stick (bishop's crook). Coll 1977 by B Barrière and subm by J M Desbordes. Estimated age: 13th century. Obazine abbey was founded AD 1130. Comment: date older than expected. Crook may have been transmitted by inheritance.

\section{Jabreilles-les-Bordes series, Haute-Vienne, lieu-dit Le Chatelard}

Study of ditch fill at foot of Historic fence (late Tene III or early Gallo-Roman period). Archael study made to determine absolute age and influence of human activities on environment.

\section{Ny-479. CH 77-1 and 1 bis}

$\mathbf{8 2 0} \pm \mathbf{8 0}$

Charcoal from excavation at back of fields under cultivation. Sample may date early ploughing. Coll by B Valadas and subm by J M Desbordes. Estimated age: 12th century. Comment: ${ }^{14} \mathrm{C}$ date confirms interpretation above.

\section{Ny-482. CH 77-6}

$940 \pm 135$

Charcoal from fill of ditch. Date may indicate surrender of ramparts and beginning of fill of ditch. Coll 1977 and subm by J M Desbordes. Estimated age: probably 10 th century. Comment: ${ }^{14} \mathrm{C}$ date agrees with expected age.

\section{Ny-480. CH 77-3 and 3 bis}

\section{AD 1275*}

$670 \pm 100$

Charcoal from fill of ditch. Coll 1977 and subm by J M Desbordes. No age expected. 
Ny-481. CH 77-5

Charcoal from top of fill of ditch. Dates end of fill of ditch. Coll 1977 and subm by J M Desbordes. Estimated age: modern. ${ }^{14} \mathrm{C}$ date agrees with expected age.

\section{E. French West Indies}

\section{Ny-500. Marie Galante Island}

$1515 \pm 85$

$\left(61^{\circ} 17^{\prime} \mathrm{W}, 15^{\circ} 50^{\prime} \mathrm{N}\right)$. Charcoal from Taliseronde site. Coll and subm by D Emond.

\section{Ny-478. La Martinique, Fond Brulé site}

$1600 \pm 250$ AD 390*

Charcoal from hearth, depth $130 \mathrm{~cm}$. Coll 1977 by M Mattoni. Site is between La Salle site (S), dated by Yale (Y-1116: $1770 \pm 80$ вP, R, 1963, v 5, p 336). Vive site (N), dated by FSU, $1730 \pm 100$ BP. Masca corrected dates are AD $190 \pm 100^{*}$ and $\mathrm{AD} 235 \pm 125^{*}$. Our sample was of poor quality, very small $(0.4173 \mathrm{~g} \mathrm{C})$. Benzene obtained was minimal, yielding large statistical error, but result seems archeologically acceptable.

\section{GEOLOGIC SAMPLES}

\section{Etang de Batéguier, Ile Ste Marguerite series, Alpes Maritimes France}

Shells from lacustrine sediments, Etang de Batéguier (5 gr 21, $9^{\prime} \mathrm{W}$, 48 gr $\left.35,8^{\prime} \mathrm{N}\right)$. Cores taken in sediments of pool, depth $2.5 \mathrm{~m}$ with level of numerous shells (Bytinidae sp, Clausilidae sp), undoubtedly killed by sudden disturbance of environment. Coll and subm 1975 by G Palausi.

\section{Ny-389. Ile Ste Marguerite}

Ny-390. Ile Ste Marguerite

Ny-391. Ile Ste Marguerite

$$
3920 \pm 90
$$$$
2530 \text { BC* }
$$

$$
1410 \pm 80
$$$$
\text { AD 580* }
$$

$$
1490 \pm 80
$$

AD 500*

General Comment (GP): Ny-389, taken near shore, is probably not significant because of possible contamination by older shells. Two dates, Ny-390 and -391, agree statistically and help estimate rate of sedimentation for pool. Mean age: $1450 \mathrm{BP}$. Rate of sedimentation: $1.80 \mathrm{~mm} / \mathrm{yr}$.

\section{SOIL SAMPLES}

\section{A. Andosols}

Andosols are soils developed on volcanic ashes. Most are mainly characterized by amorphous hydrous silica-alumina mineral such as allophanes, which can adsorb much organic matter.

Samples from Auvergne and Cantal coll 1974 by J M Hétier and B Guillet; samples from Vivarais, by J Moinereau, E N S A Montpellier. 
Puy du Mercoeur, Auvergne

$\left(45^{\circ} 42^{\prime} 12^{\prime \prime} \mathrm{N}, 2^{\circ} 55^{\prime} 12^{\prime \prime} \mathrm{E}\right)$

$\begin{array}{lll}\text { Ny-558. } & A_{12} \text { horizon, 8.8\% C, 20 to } 30 \mathrm{~cm} & 1680 \pm 80 \\ \text { Ny-559. } & B_{1} \text { horizon, 7.2\% C, 40 to } 50 \mathrm{~cm} & 3000 \pm 80 \\ \text { Ny-560. } & B_{2} \text { horizon, 3.4\% C, 60 to } 70 \mathrm{~cm} & 3700 \pm 90\end{array}$

Comment: Puy du Mercoeur is volcanic cone of basaltic cinders and scoria, S of Chaînes des Puys, 15 km SW Clermont-Ferrand, France. Volcanic eruption of Puy du Mercoeur is estimated at 4000 to $6000 \mathrm{yr}$, based on local stratigraphy (Hétier, 1975). Mean residence time of $B_{2}$ horizon organic matter probably expresses beginning of andosolization processes.

Puy de Dôme, Auvergne

$\left(45^{\circ} 45^{\prime} 25^{\prime \prime} \mathrm{N}, 2^{\circ} 55^{\prime} 14^{\prime \prime} \mathrm{E}\right)$

$\begin{array}{llr}\text { Ny-561. } & A_{1} \text { horizon, 14.2\% C, } 15 \text { to } 25 \mathrm{~cm} & 950 \pm 70 \\ \text { Ny-562. } & A_{1} B_{h} \text { horizon, 13.4\% C, 30 to } 40 \mathrm{~cm} & 2040 \pm 80 \\ \text { Ny-563. } & A_{1} B_{2} \text { horizon, 10\% C, 40 to } 50 \mathrm{~cm} & 2590 \pm 80 \\ \text { Ny-564. } & \text { II } A_{1} \text { horizon, } 1 \% \text { C, } 150 \text { to } 160 \mathrm{~cm} & 8210 \pm 120\end{array}$

Comment: mean residence time of organic matter of buried II $\mathrm{A}_{1}$ horizon agrees well with dates of charcoals separated from similar buried soils (Brousse et al, 1969). Upper ando-podzolic soil is developed on domite material $150 \mathrm{~cm}$ thick, forming of which began ca $8300 \mathrm{yr}$ BP.

Puy Mey, Auvergne

$\left(45^{\circ} 42^{\prime} 33^{\prime \prime} \mathrm{N}, 2^{\circ} 57^{\prime} 57^{\prime \prime} \mathrm{E}\right)$ Ny-565. Buried III $A_{1}$ horizon, $6 \% \mathrm{C}, 240$ to
$245 \mathrm{~cm}$

$1280 \pm 70$

Ny-566. Charcoal from buried III $A_{1}$ horizon $\quad 1510 \pm 50$

Comment: Puy Mey is a small cone on Puy du Mercoeur and Puy de la Vache, covered by unweathered volcanic scoria. Two buried andosols are observed. Organic matter of deepest, $\mathrm{Ny}-565$, had low residence time, suggesting that recovering by basaltic scoria is recent. Conclusion supported by age of charcoals, Ny-566, measured twice from 2 different samplings. If upper volcanic deposit was really caused by volcanic eruption, age of charcoals would date one of most recent volcanic events in Chaînes des Puys, ca AD 500.

Montagne de Marlieux, Cantal

$\left(45^{\circ} 13^{\prime} \mathrm{N}, 2^{\circ} 35^{\prime} 10^{\prime \prime} \mathrm{E}\right)$

$\begin{array}{llr}\text { Ny-567. } & \text { A }_{1} \text { horizon, } 12.4 \% \text { C, } 15 \text { to } 25 \mathrm{~cm} & 650 \pm 70 \\ \text { Ny-568. } & \text { B }_{1} \text { horizon, } 7.3 \% \text { C, } 40 \text { to } 50 \mathrm{~cm} & 3190 \pm 90\end{array}$ 
Ny-569. $B_{2}$ horizon, $6 \% \mathrm{C}, 60$ to $75 \mathrm{~cm}$

Comment: andosol developed on weathered material of early Pleistocene basalt, ankaramite, rejuvenated by glacial erosion. Mean residence time gradient of organic matter is very comparable to data obtained for Puy du Mercoeur andosol.

Bois des Chabottes, Vivarais $\left(44^{\circ} 40^{\prime} 23^{\prime \prime} \mathrm{N}, 4^{\circ} 21^{\prime} 10^{\prime \prime} \mathrm{E}\right)$

Ny-570. A horizon, $36 \% \mathrm{C}, 0$ to $7 \mathrm{~cm}$ undecomposed plant debris, size $>200 \mu \mathrm{m} \quad$ Modern

\section{Ny-571. A horizon, $17.4 \% \mathrm{C}, 0$ to $7 \mathrm{~cm}$} humified material, $<\mathbf{2 0 0} \mu \mathrm{m}$

$$
120 \pm 50
$$

Ny-572. B Br horizon, 8.4\% C, 7 to $25 \mathrm{~cm}$

$1340 \pm 90$

Ny-573. $\quad B_{\mathrm{h}}$ horizon, $10.2 \% \mathrm{C}, 25$ to $45 \mathrm{~cm}$

$3500 \pm 90$

Comment: this profile corresponds to evolution from andosol to podzolic soil by differentiation of ochrous $B_{i r}$ horizon (Moinereau, 1977).

General Comment: as proposed by Sharpenseel (1972), a regression curve may be established between mrt ( $\mathrm{Y}$ value in $\mathrm{yr}$ ) and depth of date samples $(\mathrm{X}$ value, in $\mathrm{cm})$. Equation for andosols $(\mathrm{Y}=60.9 \mathrm{X}+80, \mathrm{r}=0.918$, $\mathrm{n}=15$ ) suggests that gradient of mrt with depth is higher than in other soils, such as brownearths, vertisols, chernozems (Guillet, 1979). This is explained by great influence of allophanic and amorphous alumina material that biologically stabilizes adsorbed organic matter, as breakdown and turnover is delayed.

Golbey, Vosges

\section{B. Paleosols}

$\left(48^{\circ} 12^{\prime} 47^{\prime \prime} \mathrm{N}, 6^{\circ} 26^{\prime} 12^{\prime \prime} \mathrm{E}\right)$. Surface of old fluvial terrace of Moselle was covered by loam, $1.40 \mathrm{~m}$ thick, on which sol lessivé glossique (agric glossaqualf) is differentiated. Old surface corresponds to cryogenic humic soil with charcoals. Samples coll and subm 1976 by M Cailler, CPB Nancy.

Ny-588. Humic paleosol, $<\mathbf{5 0 0} \mu \mathbf{m}, \mathbf{1 . 8 \%}$ C,
140 to $150 \mathrm{~cm}$


and of humic acid fraction of charcoals was probably caused by continuous percolation of organic matter and water through cracks of upper loamy soil.

\section{Le Bernardan, Haute Vienne}

$\left(46^{\circ} 19^{\prime} 18^{\prime \prime} \mathrm{N}, 1^{\circ} 14^{\prime} 41^{\prime \prime} \mathrm{E}\right)$ Indurated iron pans in arenaceous granitic parent material coll and subm 1975 by J C Flageollet, Univ Nancy II.

\section{Ny-586. Le Bernardan I, 0.3\% C, 30 to $40 \mathrm{~cm} \quad 2230 \pm 80$ \\ Ny-587. Le Bernardan II, 0.1\% C, 80 to $90 \mathrm{~cm} \quad 15,690 \pm 430$}

Comment: for deepest pan, older age was expected. Rejuvenation by root decomposition is possible.

\section{Soil organic matter fractionation}

Various fractions of $A_{1}$ and $B_{1}$ horizons of Puy du Mercoeur andosol were processed to determine different turnover rates of organic matter fractions. Soil organic matter extractions were performed with successive $\mathrm{Na}$-pyrophosphate $0.1 \mathrm{M}$ and $\mathrm{NaOH} \mathrm{N}$ solutions. Fulvic acids are soluble when alkaline organic solutions are acidified $(\mathrm{pH}$ l) causing humic acids to precipitate. Humine is non-extractable organic matter. Values in brackets report organic matter extracted as Carbon percent of total $\mathrm{C}$ of soil sample.

Puy du Mercoeur, $A_{1}$ horizon

Ny-574. Fulvic acids 1, (15)

$1330 \pm 70$

Precipitated at $\mathrm{pH} 4.8: \beta$ humus.

Ny-575. Fulvic acids 2, (13)

$650 \pm 70$

Fulvic acid fraction that does not precipitate at $\mathrm{pH} 4.8$.

Ny-576. Humic acids, (13)

$1510 \pm 70$

Ny-577. Humine, (45)

$1460 \pm 70$

Comment: except for Fulvic acids 2, mean residence times of humus $-\mathrm{C}$ fractions are very similar.

Puy du Mercoeur, $B_{1}$ horizon

Ny-578. Fulvic acids 1 (11)

$2580 \pm 80$

Ny-579. Fulvic acids 2 (12.4)

$2130 \pm 80$

Ny-580. Humic acids (17.5)

$2760 \pm 80$

Ny-581. Humic acids: acid hydrolyzate (3.5)

$1830 \pm 80$

Ny-582. Humic acids: hydrolyzate residue (14)

$3270 \pm 80$

Ny-583. Humine fraction (55.5)

$2870 \pm 80$

Ny-584. Humine: acid hydrolyzate (27)

$2340 \pm 80$ 
Ny-585. Humine: hydrolyzate residue (29.5) $\quad 3180 \pm 80$

Comment: as expected, hydrolyzate fractions of humic acids and humine are relatively more renewed than residual carbon fractions. This may be considered a constant characteristic of soil organic chemistry, resulting from higher turnover rate of aminopolysaccharides and other metabolic $\mathrm{N}$-products, which may be liberated from humic polycondensates by $6 \mathrm{~N}$ $\mathrm{HCl}$ hydrolysis procedure for $16 \mathrm{hrs}$.

\section{REFERENCES}

Brousse, R, Delibrias, G, Labeyrie, J, and Rudel, A, 1969, Eléments de chronologie des éruptions de la Chaîne des Puys: Soc Géol France Bull, v 7, no. 11, p 770-793.

Francfort, H P and Pottier, M H, 1978, Sondage préliminaire sur l'établissement protohistorique harappéen et post-harappéen de Shortugaï (Afghanistan du NE): Arts Asiatiques 1978, v 34, p 29-79.

Guillet, B, 1979, Etude du renouvellement des matières organiques des sols par les radioisotopes $\left({ }^{14} \mathrm{C}\right)$ en Pédologie, constituants et propriétés: Paris, Masson éd, p 210 226.

Hassko, B, Guillet B, Jaegy R, and Coppens R, 1974, Nancy natural radiocarbon measurements III: Radiocarbon, v 16, no. 1, p 118-130.

Hétier, J M, 1975, Formation et évolution des andosols en climat tempéré: Thesis, Univ Nancy, $194 \mathrm{p}$.

Moinereau, J, 1977, Altération des roches, formation et évolution des sols sur basalte sous climat tempéré humide (Velay-Vivarais-Coirons): Thesis, Univ Montpellier, $139 \mathrm{p}$.

Ralph, E K, Michael, H N, and Han, M C, 1973, Radiocarbon dates and reality: Masca Newsletter, v 9, no. 1 .

Sharpenseel H W, 1972, Natural radiocarbon measurement of soil and organic matter fractions and on soil profiles of different pedogenesis: Internatl conf on radiocarbon dating, 8th Proc, Wellington, New Zealand, E, p 3-11. 